\title{
CARACTERÍSTICAS GENOTÍPICAS E FENOTÍPICAS EM ATLETAS VELOCISTAS
}

\author{
CHARACTERISTICS GENOTIPICS AND FENOTIPICS \\ IN ATHLETICS SPRINTERS
}

\begin{abstract}
RESUMO
AUTORES

Leonardo Chrysostomo dos Santos ${ }^{1}$ Paulo Moreira Silva Dantas ${ }^{1,2}$

José Fernandes Filho"

${ }^{1}$ PROCIMH - UCB/RJ

- Programa de Mestrado

em Ciência da Motricidade Humana

${ }^{2}$ UNIGRANRIO/RJ

CARACTERISTICAS GENOTÍPICAS

E FENOTÍPICAS EM

ATLETAS VELOCISTAS

4(1): 49-56

PALAVRAS-CHAVE

dermatoglifia; somatotipo;

isocinético; corredores de velocidade.

\section{KEYWORDS}

dermatoglyphic; somatotype;

isokinetic; runners sprinters.

data de submissão

Julho 2007

data de aceitação

Novembro 2007

O objetivo deste estudo foi comparar
as características genotípicas e fenotípicas em um grupo de atletas de velocidade no atletismo. Fizeram parte do estudo 19 atletas de ambos os sexos, descritos pela idade de $22,42 \pm 3,53$, massa corporal de $66,61 \pm 7,66 \mathrm{~kg} \mathrm{e}$, estatura de $173,18 \pm 7,23 \mathrm{~cm}$. 0 perfil genético e o somatotipo foram estimados com base no protocolo de Cummins e Midlo (1961), e Heath e Carter (1990), respectivamente, e a avaliação isocinética referenciada em Adams (1998). Os resultados demonstram que, no grupo: (a) $D 10=13$ e $S Q T L=$ 120 , (b) para os tipos de desenhos, $A=2,1 \%$; $L=64,7 \%$ e $W=33,2 \%$, (c) o perfil foi classificado por mesomorfo-balanceado sendo os valores 2,33 - 4,11 - 2,81, (d) os valores sugeridos para o torque isocinético nos diferentes intervalos entre as repetições 1-3 e 48-50 foram, respectivamente, $348,67 \pm 24,4 \%$ e, $150 \pm 24,7 \%$, (e) não existe diferença significativa para um valor de $p<0,0001$ entre a classificação das variáveis genotípicas e fenotípicas. Em conclusão, este estudo acumula a possibilidade de inserção da dermatoglifia, neste âmbito esportivo, como método prognóstico que pode auxiliar, no barateamento e, na avaliação diagnóstica do sujeito.
\end{abstract}

ABSTRACT
The purpose this study was to comparate the genotipics and the phenotipics characteristics in a group of the runners sprint. They were part 19 athletes of both sexes and describe for 22,42 $\pm 3,53$ age, 66,61 \pm $7,66 \mathrm{~kg}$ body mass and 173,18 \pm $7,23 \mathrm{~cm}$ weight. The genetic profile and the somatotype were based in Cummins \& Midlo (1961) and Heath \& Carter (1990) respectively, and the evaluation isokinetic were based in Adams (1998). The found results that, in group: (a) $D 10=12,00$ and $S Q T L=123$, (b) for the types of drawings, $A=2,1 \%$; $L=64,7 \%$ and $W$ $=33,2 \%$, (c) the profile somatotypic of the group was classificated by meso-balance, the values was 2,33 - 4,11 - 2,81, (d) the values suggested for the torque isokinetic in the different intervals among the repetitions 1-3 and 48-50, were respectively, $348,67 \pm 24,4 \%$ and, $150 \pm$ 24,7\%, (e) doesn't exist significant difference for a value of $p<0,0001$ between the classification of the variables genotipics and fenotipics. In conclusion, it study accumulates the possibility dermatoglyphics insert in this sporting, as method prognostic that can aid, in the lower cost and, in the subject's evaluation diagnostic. 


\section{INTRODUC̣ÃO}

Vale ressaltar por oportuno que, no âmbito das modalidades esportivas, o perfil dermatoglífico tem sido investigado e, para tanto, cabe citar as pesquisas envolvendo a seleção brasileira de ginástica olímpica, praticantes de basquetebol, atletas de voleibol brasileiro masculino adulto, atletas de futsal brasileiro masculino adulto, nadadores meio-fundistas e fundistas de alto rendimento, atletas brasileiros de corrida de orientação de alto rendimento, a seleção brasileira de futebol de praia, ciclistas de alto rendimento e a seleção brasileira feminina adulta de andebol $^{8,10,21,22,24,25,26,27,31}$.

Condizente a utilização do método isocinético, cabe apresentar algumas de suas possibilidades de aplicação que vão desde investigações acerca da força e equilíbrio muscular de atletas, até estudos de revisão bibliográfica sobre os aspectos práticos da avaliação isocinética e sua utilização para os indivíduos que praticam atividade física ${ }^{5,6,7}$.

Faz-se pertinente ressaltar que a classificação somatotípica é empregada com o objetivo de: (1) descrição e comparação, entre desportistas de distintos níveis; (2) caracterização das mudanças no físico durante o crescimento, o envelhecimento e o treinamento; (3) comparação da forma relativa de jogadores masculinos e femininos; (4) aplicação como ferramenta para a análise da imagem corporal $^{8,11,14,15,16,29}$. Sobretudo, sabe-se que consta no acervo do conhecimento científico que a seleção dos esportistas, para diferentes modalidades de jogos, se apóia nas respectivas capacidades de resolver tarefas motoras, de caráter tático, de maneira eficiente, e, que ao dispor-se de uma metodologia de marcas genéticas, se evidencia não a ausência dessas variáveis intervenientes, mas o aprimoramento de um processo que aumentaria a certeza de manter sob a mira os indivíduos que já possuam um genótipo para esta ou aquela modalidade, ou ainda, provas desportivas $^{31}$.

Portanto, é pressuposto, aqui, que uma das condições necessárias para se conseguir sucesso dentro do contexto de alto rendimento esportivo, da Educação Física, é o talento inato ou a aptidão do atleta. Cabendo, também, destacar que a utilização de informações prévias acerca das capacidades e das tendências genéticas aliadas à contribuição fenotípica possibilita tanto a determinação de um talento quanto com seu desenvolvimento. Neste contexto, é possível constatar a carência de estudos que envolvam atletas de corrida de velocidade.

Contudo, faz-se necessário observar que a aplicabilidade prática da dermatoglifia e da somatotipia no processo de desenvolvimento das estratégias de intervenção profissional, com cunho de orientação e de seleção desportiva, demonstrou que o meio (fenótipo), no qual os sujeitos observados habitam, acarretou em influências positivas ou negativas, na confirmação dos dados extrapolados. Sobretudo, recomendaram a realização de futuras investigações que busquem estabelecer uma associação entre a avaliação do estado (fenótipo) e o potencial genético (dermatoglifia) $^{14}$.

Portanto, o objetivo geral desse estudo centra-se em comparar as características genotípicas e fenotípicas em atletas velocistas da modalidade de atletismo, de ambos sexos e da categoria adulta, referenciado pelos protocolos de dermatoglifia $^{20}$, somatotipia ${ }^{30} \mathrm{e}$, isocinético ${ }^{3}$.

\section{METODOLOGIA}

A amostra foi selecionada a partir de um grupo de atletas de corrida de velocidade ranqueados pela Confederação Brasileira de Atletismo $^{33}$ e, composta por 19 atletas de alto rendimento e de ambos os sexos descritos pela idade de $22,42 \pm 3,53$ anos, massa corporal de $66,71 \pm 7,66$ quilogramas $[\mathrm{kg}$ ] e, estatura de 173,18 \pm 7,23 centímetros (cm).

No que diz respeito aos procedimentos metodológicos, cabe destacar que num primeiro momento todos os indivíduos assinaram um termo de consentimento livre e esclarecido, e também preencheram os dados solicitados na ananmese. Cabendo destacar que o presente trabalho atendeu às Normas para a Realização de Pesquisa em Seres Humanos, resolução 196/96, do Conselho Nacional de Saúde de 10/10/1996 e foi aprovado pelo Comitê de Ética, em Pesquisa Envolvendo Seres Humanos da Universidade Castelo Branco.

0 perfil dermatoglífico foi referenciado pelo protocolo de dermatoglifia ${ }^{20}$, para o qual, na coleta das impressões digitais, utilizou-se papel no formato A4 e o coletor de impressões digitais da marca Impress ${ }^{\circledR}$.

Já as características somatotípicas foram avaliadas pelo método somatotipológico ${ }^{30}$ que permite um estudo apurado sobre o tipo físico ideal de cada modalidade esportiva. Para tanto recorreu-se a seguinte instrumentação: (1) uma balança de marca Filizola, devidamente calibrada e aferida, com capacidade de aferição entre de zero a $150 \mathrm{~kg}$ e precisão de 100 gramas. (2) um adipômetro científico, do tipo Harpenden marca Cescorf, cuja precisão é de um milímetro. (3) um estadiômetro, construído de madeira da Cardiomed Ltda. (4) uma fita mé- 
trica de metal flexível, do fabricante American Medical do Brasil Ltda. marca Sanny, com dois metros de comprimento e precisão de um milímetro. (5) um paquímetro da marca Rosscraft, modelo Tommy 02 (made in Canadá), cuja variação é de dois a $18 \mathrm{~cm}$, com precisão de um milímetro.

Para o protocolo de avaliação isocinética $^{3}$ foi utilizado um aparelho isocinético de extensão unilateral do joelho, devidamente calibrado e, da marca Kin-Com - fabricante Chattanoga Group ${ }^{\circledR}$. A velocidade e as repetições estipuladas foram de $180^{\circ} . s^{-1}$ e 50 , respectivamente. Antes da execução de cada fase deste protocolo, os indivíduos realizaram cinco repetições completas do movimento, com o mínimo esforço possível, a fim de familiarizarem-se com o equipamento. Por fim, há de se destacar que o estimulo verbal foi fornecido a cada sujeito com o objetivo de produzir contrações voluntárias máximas durante o teste.

0 tratamento estatístico atendeu à proposta básica, utilizando a estatística descritiva com o intuito de constituir o perfil de cada indivíduo do grupo, visando caracterizar o universo amostral pesquisado, para obtenção das variáveis de natureza discreta. Utilizaram-se as distribuições de freqüência, quanto às de natureza contínua, isto é, aquelas que obedeceram a um sistema métrico bem definido e normalizado, em que foram seguidos os parâmetros estatísticos básicos, como: tamanho da população (N), média (X), desvio padrão (s); mediana (Med), valores mínimos e máximos $^{18}$.

No sentido de aumentar a potência dos resultados e garantir a confiabilidade dos mesmos foi aplicado o teste Não-Paramétrico de Normalidade, Komogorov-Smirnov.

\begin{tabular}{|c|c|c|c|c|c|c|}
\hline Sexo & & A & $\mathbf{L}$ & $\mathbf{w}$ & D10 & SQTL \\
\hline \multirow{6}{*}{ Feminino } & $\mathbf{N}$ & 5 & 5 & 5 & 5 & 5 \\
\hline & $x$ & $0 \%$ & $68 \%$ & $32 \%$ & 13,2 & 104,2 \\
\hline & $\mathbf{s}$ & 0 & 2,77 & 2,77 & 2,77 & 27,27 \\
\hline & Med & 0 & 8 & 2 & 12 & 105 \\
\hline & Mínimo & 0 & 3 & 0 & 10 & 60 \\
\hline & Máxima & 5 & 10 & 7 & 17 & 130 \\
\hline \multirow{6}{*}{ Masculino } & $\mathbf{N}$ & 14 & 14 & 14 & 14 & 14 \\
\hline & $x$ & $0,29 \%$ & $6,36 \%$ & $3,36 \%$ & 13,07 & 126,07 \\
\hline & s & 0,61 & 2,59 & 2,79 & 3,1 & 31,71 \\
\hline & Med & 0 & 6,5 & 3 & 13 & 130 \\
\hline & Mínimo & 0 & 0 & 0 & 9 & 78 \\
\hline & Máxima & 2 & 10 & 10 & 20 & 202 \\
\hline \multirow{7}{*}{ Total } & $\mathbf{N}$ & 19 & 19 & 19 & 19 & 19 \\
\hline & $x$ & $0,21 \%$ & $6,47 \%$ & $3,32 \%$ & 13,11 & 120,32 \\
\hline & s & 0,54 & 2,57 & 2,71 & 2,94 & 31,46 \\
\hline & Med & 0 & 7 & 2 & 12 & 123 \\
\hline & Mínimo & 0 & 0 & 0 & 9 & 60 \\
\hline & Máxima & 2 & 10 & 10 & 20 & 202 \\
\hline & & Valor & e Tendên & Central da & ariáveis c & $\begin{array}{r}\text { TABELA1 } \\
\text { latoglificas }\end{array}$ \\
\hline
\end{tabular}

Ainda na tentativa de melhor elucidar as relações existentes entre as variáveis foi utilizado o método estatístico, de estudo da proporcionalidade $^{23,32}$, para o qual, na tentativa de transformar os valores em um único intervalo foi utilizada a seguinte equação: (valor observado -menor valor observado)/(maior valor observado-menor valor observado].

A estatística inferencial que compõe o teste de hipóteses foi baseada na classificação, para a qual foi feito um cruzamento comparativo entre as classificações, segundo o genótipo e fenótipo, utilizando-se o teste $U$ de Mann-Whitney para variáveis categóricas ordinais ${ }^{18}$.

Por fim, e objetivando-se a medição dos testes, o presente trabalho se pautou em consonância às considerações básicas do tratamento estatístico, a fim de manter-se a cientificidade da pesquisa, em que se considerou o nível de significância de $\mathrm{p}<0,05$, isto é, 95\% de probabilidade para as afirmativas e/ou negativas, denotadas durante as investigações ${ }^{1}$.

\section{RESULTADOS}

Pode-se observar na tabela 1 os valores de tendência central de média $(X)$, desvio padrão (s), mediana (Méd) e amplitude (mínimo e Maximo] das variáveis dermatoglificas do Arco (A), Presolha (L), Verticilo (W) Delta 10 (D10) e Somatório total da quantidade de linhas (SQTL). Após a verifiação da normalidade destas variáveis através do teste de KomogorovSmirnov verificou-se que o arco é o único dado não paramétrico. 


\begin{tabular}{llccc}
\hline Sexo & & Endomorfia & Mesomorfia & Ectomorfia \\
\hline \multirow{4}{*}{ Feminino } & $\mathrm{N}$ & 5 & 5 & 5 \\
& $\mathrm{X}$ & 2,25 & 3,35 & 3,64 \\
& $\mathrm{~s}$ & 1,02 & 1,46 & 1,43 \\
& Med & 2,61 & 4,25 & 4,14 \\
& Mínimo & 1,15 & 1,15 & 1,16 \\
& Máxima & 3,24 & 4,55 & 4,65 \\
\hline \multirow{4}{*}{ Masculino } & $\mathrm{N}$ & 14 & 14 & 14 \\
& $\mathrm{~N}$ & 2,35 & 4,38 & 2,52 \\
& $\mathrm{X}$ & 0,73 & 0,88 & 0,95 \\
& Med & 2,33 & 4,1 & 2,75 \\
& Mínimo & 1,31 & 3,32 & 0,45 \\
& Máxima & 4,2 & 6,22 & 4,22 \\
\hline \multirow{4}{*}{ Total } & $\mathrm{N}$ & 19 & 19 & 19 \\
& $\mathrm{X}$ & 2,33 & 4,11 & 2,81 \\
& $\mathrm{~s}$ & 0,79 & 1,12 & 1,17 \\
& Med & 2,36 & 4,11 & 2,82 \\
& Mínimo & 1,15 & 1,15 & 0,45 \\
& Máxima & 4,2 & 6,22 & 4,65 \\
\hline
\end{tabular}

TABELA2

Valores de Tendência Central das variáveis somatotipicas.

\begin{tabular}{llccc} 
Sexo & & Iso_início & Iso_Fim & $\% \mathbf{Q}$ \\
\hline \multirow{4}{*}{ Feminino } & $\mathrm{N}$ & 5 & 5 & 5 \\
& $\mathrm{X}$ & 254,13 & 111,6 & 44,74 \\
& $\mathrm{~S}$ & 59,26 & 17,73 & 5,78 \\
& Med & 247,67 & 112 & 45,2 \\
& Mínimo & 185,33 & 90 & 35,1 \\
& Máxima & 343 & 135,67 & 49,8 \\
\hline \multirow{4}{*}{ Masculino } & 14 & 14 & 14 \\
& $\mathrm{~N}$ & 386,41 & 162,02 & 42,47 \\
& $\mathrm{X}$ & 64,41 & 32,43 & 8,46 \\
& S & 372 & 158,17 & 44,45 \\
& Med & 294 & 97,33 & 29,5 \\
& Mínimo & 227,67 & 53 \\
\hline \multirow{4}{*}{ Total } & Máxima & 506,67 & 19 & 19 \\
& N & 19 & 148,75 & 43,07 \\
& X & 351,6 & 36,74 & 7,76 \\
& S & 85,78 & 150 & 45,2 \\
& Med & 348,67 & 90 & 29,5 \\
& Mínimo & 185,33 & 227,67 & 53
\end{tabular}

TABELA3

Valores de Tendência Central das variáveis isocinéticas.
Pode-se observar na tabela 2 os valores de tendência central de média (X), desvio padrão (s), mediana (Méd) e amplitude (mínimo e Maximo] das variáveis. Somatotipicas: Endomorfia; Mesomorfia e Ectomorfia.

Pode-se observar na tabela 3 os valores de tendência central de média (X), desvio padrão (s), mediana (Méd) e amplitude (mínimo e Maximo] das variáveis.isocinéticas Isocinético nas três repetições iniciais (Iso_início), Isocinético nas três últimas repetičões (Iso_Fim) e Percentual de Queda entre Ambas [\%Q].

Pode-se observar na tabela 4 os valores de tendência central de média (X), desvio padrão (s), mediana (Méd) e amplitude (mínimo e Maximo] das variáveis Fenotipicas e Genotipicas.

Pode-se observar na figura 1 (grupo feminino $n=5$ ) e na figura 2 (grupo masculino $n=14$ ] os resultados da proporcionalidade estatística das variaveis isocinéticas, somatotipicas e dermatoglificas.

\section{DISCUSSÃO}

É importante notar que a tabela 1 apresenta os valores referentes ao perfil dermatoglífico dos atletas de corrida de velocidade, do presente estudo. Contudo, cabe ressaltar que a alta variabilidade existente intragrupo, no que diz respeito à quantidade de deltas em dez dedos (D10) e ao somatório da quantidade total de linhas (SQTL), caracteriza uma falta de normalidade.

Isto posto, ilustrando a argumentação contida nos autores ${ }^{21}$ que creditaram ser comum, no processo de seleção dos atletas brasileiros, o critério de escolha 
considerar, predominantemente, o estado físico e técnico dos atletas em detrimento a preocupação com o potencial genético apresentado por eles. Tornando, portanto, indispensáveis às investigações e explicações acerca do processo genético e suas possibilidades de auxiliar o treinamento desportivo, objetivando potencializar as probabilidades destes atletas chegarem a um melhor desempenho, relacionando-os com a modalidade desportiva.

Contudo, destaca-se que o presente grupo apresenta um perfil de qualidades físicas referentes à forca relativa e absoluta, e à estatura ${ }^{30}$. Ainda assim, e tendo por base 0 resultado observado em $L>W$, é possivel constatar que ambos os sexos apresentaram características genótipicas de capacidade glicolítica e também anaeróbica alática, sendo que o grupo masculino apresentou mais características glicolíticas que o grupo feminino, constatado, também, por uma maior presença de SQTL, que é um indicativo de maior endurance $e^{9,12,13}$. Concernente à análise dos desenhos digitais, ressalta-se que estes apontam para o aumento na complexidade dos desenhos, e corroboram com os autores que observaram uma tendência ao desaparecimento do arco e ao aumento dos desenhos mais complexos no alto rendimento esportivo ${ }^{2,26,29}$.

Com base nos resultados apresentados na tabela 2, cabe destacar que o grupo feminino foi classificado como ectomorfo-mesomorfo e, o grupo masculino apresentou uma classificação de mesomorfo-balanceado, assim como a classificação geral do grupo.

Em diferentes contextos e convergindo com os dados do presente estudo, também foram identificadas as características somatotípicas

\begin{tabular}{llcc}
\hline Sexo & & Fenótipo & Genótipo \\
\hline \multirow{4}{*}{ Feminino } & $\mathrm{N}$ & 5 & 5 \\
& $\mathrm{X}$ & 3 & 3 \\
& $\mathrm{~S}$ & 1,58 & 1,58 \\
& Med & 3 & 3 \\
& Mínimo & 1 & 1 \\
& Máxima & 5 & 5 \\
\hline \multirow{4}{*}{ Masculino } & $\mathbf{N}$ & 14 & 14 \\
& $\mathrm{~N}$ & 7,5 & 7,5 \\
& $\mathrm{X}$ & 4,18 & 4,18 \\
& $\mathrm{~S}$ & 7,5 & 7,5 \\
& Med & 1 & 1 \\
& Mínimo & 14 & 14 \\
\hline \multirow{4}{*}{ Total } & Máxima & 19 & 19 \\
& $\mathrm{~N}$ & 6,32 & 6,32 \\
& $\mathrm{X}$ & 4,16 & 4,16 \\
& $\mathrm{~S}$ & 5 & 5 \\
& Med & 1 & 1 \\
& Mínimo & 14 & 14 \\
& Máxima & & \\
& & & TABELA4 \\
& & Valores de Tendência Central das variáveis: & Fenótipo e Genótipo. \\
& & &
\end{tabular}

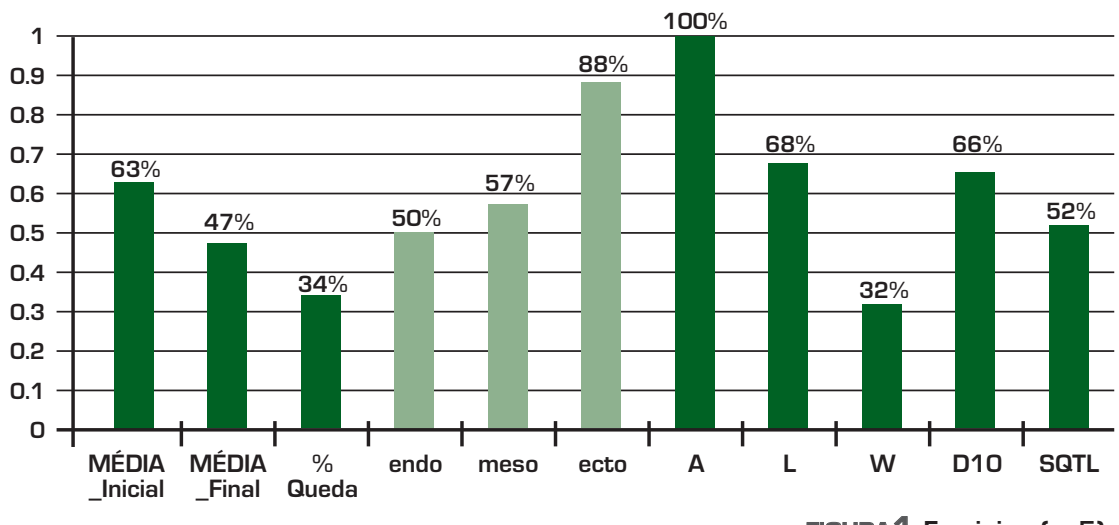

FIGURA1 Feminino $(n=5)$.

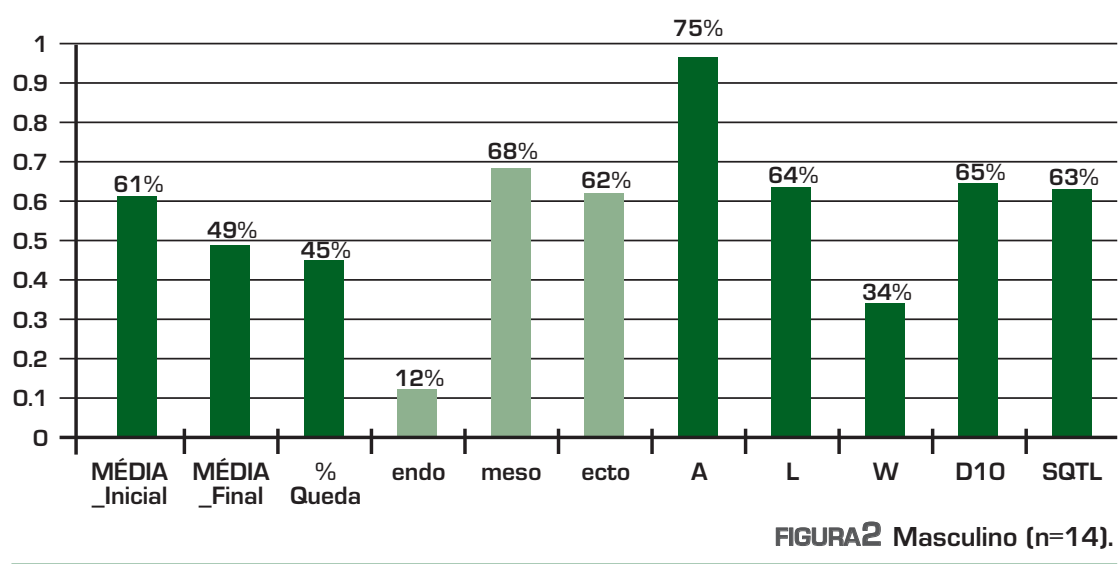


da seleção brasileira de handebol feminino $^{22}$ e foram atribuídas predominâncias do componente mesomorfo, assim como em ciclistas de alto rendimento ${ }^{6}$, e em atletas de corrida de orientação ${ }^{27}$. Sobretudo, em atletas brasileiros de corrida de resistência ${ }^{16}$, foi identificada a predominância de um perfil ectomorfo-mesomorfo, fato que converge com os presentes dados no que diz respeito ao perfil somatotípico feminino, mas diverge quanto à modalidade esportiva.

No que se refere aos resultados demonstrados na tabela 3 , cabe observar que, no grupo feminino, houve uma queda acentuada para as três últimas repetições, no teste isocinético, sugerindo que as atletas em questão possuem uma maior capacidade de gerar grandes torques do que uma capacidade de manutenção do mesmo. Isto posto, é possivel creditar que estas atletas têm uma grande capacidade de velocidade máxima e de aceleração e que, muito embora a queda do torque seja esperada, por serem atletas de provas de velocidade explosiva, poder-se-ia sugerir que quanto menor for o percentual de queda mais interessante é a capacidade de manutenção de força e velocidade. Em contrapartida, o grupo masculino apresentou uma queda menos acentuada nos torque das três ultimas repetições, sugerindo um comportamento de maior manutenção da força e da velocidade, conforme esclarecido anteriormente.

llustrando e fundamentando a informação contida no parágrafo anterior, cabe apresentar especialmente, o estudo da relação entre os índices dermatoglíficos e o resultado de uma avaliação isocinética e de ergoespirometria, em jogadores profissionais de futebol de campo ${ }^{4}$. Nele, os sujeitos foram divididos em dois grupos, para o qual o critério estabelecido foi apresentar uma porcentagem de $70 \%$ de $\mathrm{W}$ ou $\mathrm{L}$. Os resultados após a avaliação isocinética indicaram que o grupo que possuía $70 \%$ de $W$, iniciou o exercício com uma produção menor de torque, mas conseguiu manter um melhor padrão de torque até o final da tarefa, quando comparados ao grupo que possuía $70 \%$ de L. Corroborando com os presentes dados que demonstram um percentual de $L=64,74 \%$ aliados a uma redução de mais de $50 \%$ entre os valores médios dos intervalos de repetições.

Há de se destacar que a maior endurance genotípica observada no grupo masculino, associada a maior quantidade de SQTL apresentada, corroboram com os valores inferiores de queda das três primeiras repetições com as três últimas, no teste isocinético. Por outro lado, o autor do iente estudo chama a atenção ao comportamento de queda mais abrupto no grupo feminino que se coaduna com um genótipo mais característico de coordenação neuromotora e menos acentuado de coordenação de endurance demonstrado pelo D10 e SQTL ${ }^{31,32}$.

Os dados demonstrados na tabela 4 e nas figuras 1 e 2 indicam, pelo estudo de proporcionalidade, a relação existente entre o fenótipo e genótipo onde o genótipo tanto do grupo masculino quanto do grupo feminino está manifestado no fenótipo. Entretanto, com base no resultado do teste de hipótese, o presente estudo aceita a hipótese nula. Em outros termos, não existe diferença significativa para um valor de $p<0,0001$ entre a classificação das variáveis genotípicas e fenotípicas.
Diante do exposto, cabe destacar, sob a identificação de características genéticas, que a interligação (pontos em comum] entre "as Impressões Digitais - a modalidade esportiva - a posição esportiva" reflete a lei natural biológica geral das ligacõoes mútuas das marcas genéticas com as aptidões congênitas das manifestações funcionais independentemente da pertinência àquela população. Por conseguinte, é cabivel acreditar que as Impressões Digitais podem ser utilizadas como critérios de orientação esportiva precoce e da seleção esportiva nas condições do Brasil e de qualquer outro País ${ }^{1}$.

Por outro lado, também, destaca-se a opinião dos autores que defendem que a dermatoglifia, como forma de predizer a performance motora, não se demonstrou um método eficiente ${ }^{19}$. Com isso, paralelamente, sugere-se novas investigações que incluam, em sua amostra, indivíduos que não sejam atletas e que possuam um perfil dermatoglífico, supostamente, favorável a uma determinada capacidade física específica de um desporto, obviamente, analisando as respostas geradas após um treinamento.

É importante notar que os resultados apresentados no iente estudo constituem valores interessantes, que podem e devem ser usados, também, como forma de avaliação do prognóstico de eficiência da prática destes e de outros atletas de corrida de velocidade.

Em última análise, vale ressaltar por oportuno que o perfil dermatoglífico do presente grupo, conforme esperado pelas particularidades da corrida de velocidade ${ }^{34}$, divergiu do perfil observado no estudo em atletas de corrida de resistência ${ }^{16}$. Entretanto, com base no resultado do mesmo estudo, o perfil somatotípico dos atletas tem predominância 
igual ao do presente estudo. Resultado este, não interessante e que pode ser atrelado à variabilidade de D10 e SQTL do presente grupo e, conseqüentemente, ilustrando a maior relação entre dermatoglifia e isocinético.

Sobretudo, o presente estudo buscou contribuir para uma elucidação de possibilidades oriundas da interligação entre as características genotípicas e fenotípicas, mostrando que o conhecimento acerca desta relação é de suma importância para o crescimento e desenvolvimento dos processos que envolvem o treinamento desportivo. Processos estes, que podem auxiliar desde a otimização na identificação das cargas genéticas e/ou fenotípicas até o barateamento dos custos envolvidos, em detrimento da escolha de um teste sobre o outro. Outrossim, acumulando a possibilidade da inserção da dermatoglifia, neste âmbito esportivo, como método prognóstico que pode auxiliar na avaliação diagnóstica do sujeito.

\section{CORRESPONDÊNCIA}

Leonardo Chrysostomo dos Santos

E-mail:

leochrysostomo@terra.com.br

\section{REFERÊNCIAS}

1. Abramova TF, Chafranova El, Nikitina TM (1995). Impressões dermatoglíficas - marcas genéticas na seleção nos tipos de esporte / atualidades na preparação de atletas nos esportes cíclicos. Coletânea de artigos científicos. Volvograd, 86-91.
2. Abramova TF, Nikitina TM, Ozolin NN (1996). Impressões dermatoglíficas - marcas genéticas no potencial energético do homem. Anais Científicos de Moscou. Moscou, 3-13.

3. Adams GM (1998). Exercise Physiology Laboratory Manual. California: WCB Mc Grawl-Hill.

4. Almeida MN, Dantas PMS, Fernandes Filho J (2005). Relações dos índices dermatoglíficos com avaliação isocinética e ergoespirometria. F\&PJ 4:101-106.

5. Amatuzzi MM, Greve JMD, Terreri ASAP (2001). Avaliação isocinética no joelho de atleta. Rev Bras Med Esp 7:170-174.

6. Andrade MS, Fleury AM, Silva AC (2005). Força muscular isocinética de jogadores de futebol da seleção paraolímpica brasileira de portadores de paralisia cerebral. Rev Bras Med Esp 11:281-285.

7. Ascensão A, Carvalho J, MagaIhães J, Mota J, Oliveira J, Soares JMC (2003). Efeito de um programa de treino em idosos: comparação da avaliação isocinética e isotônica. Rev Paul Ed Fís 17:74-84.

8. Assis M, Dantas PMS, Fazolo E, Fernandes Filho J, Tuche W (2005). Perfil dermatoglífico e somatotípico de ciclistas de alto rendimento do Brasil. Revista de Educação Física 132:14-19.

9. Bogdanov OA, Komisova VY, Kuranev VS (2006). Comparative of Physical Development and Physical Preparedness of Female Students, who Entered Gertsen's RSPU in 1983 and 2005. Teoria I Praktika Fiziceskoi Kul'tury 9:55-56.

10. Borin JP. (2002) Utilização da discriminação gráfica de Fisher para indicação dos dermatóglifos como referencial de potencialidade de atletas de basquetebol. Tese (Doutorado em Educação Física). Universidade Estadual de Campinas, Campinas, 2002.
11. Borms, J, Carter, JE, Hebbelinck, Ross WD (1980). Anthropometric characteristics of female Olympic rowers. Can J Appl Sport Sci 5:255-262.

12. Butova OA, Butov VS (2003). Individual variations of the immune status in relation to the types of body-build in 15-year-old boys living in the cities of the southern part of Russia. Papers on Anthropology 12:42-50.

13. Butova OA, Lisova IM (2001). Correlations of various parameters of the human constitution. Morfologiia v. 119, n. 2, pp. 63-66.

14. Cardoso PG, Costa G, Dantas PMS, Fazolo E, Fernandes Filho J, Menezes IC, Nunes RMA, Portal MND, Teixeira MES, Tuche W (2005). A dermatoglifia e a somatotipologia no alto rendimento do beach soccer - seleção brasileira. Revista de Educação Física 130: 45-51.

15. Carter JE (1970). The somatotypes of athletes - a review. Hum Biol 42:535-569.

16. Carvalho E, Fernandes Filho J, Novaes JS (2005). Perfis dermatoglífico, somatotípico e fisiológico de atletas de alto rendimento, participantes de corrida de resistência, no Rio de Janeiro. F\&PJ 4:168-174.

17. Casagrande G, Viviani F (1993). Somatotype of Italian rugby players. J Sports Med Phys Fitness 33:65-69.

18. Costa Neto PLO (2002). Estatística. São Paulo: Edgard Blücher.

19. Costa PIAM, Gentil P, Junior VAR, Lobato SRU, Nassau FF (2006). Correlação entre perfil datiloscópico e performance nos testes de 12 minutos e de impulsão horizontal em jovens do sexo masculino. Revista Digital de Buenos Aires ano 11:100, Setembro. 
20. Cummins H, Midlo CH (1961). Finger prints, palmes and soles: an introduction to dermatoglyphics. New York: Dover Publications.

21. Cunha Junior AT, Dantas PMS, Fernandes Filho J, Nogueira T (2005). Perfil somatotípico, dermatoglífico e das qualidades físicas da seleção brasileira de handebol feminino adulto por posição de jogo. F\&PJ 4:236-241.

22. Cunha ACPT, Cunha Junior AT, Dantas PMS, Schneider AT (2006). Características dermatoglíficas, somatotípicas, psicológicas e fisiológicas da seleção brasileira feminina adulta de handebol. F\&PJ 5 : 81-86.

23. Díaz FR, López FGB (2007). Bioestatística. São Paulo: Thomson Pioneira.

24. Fernandes Filho J, Medina MF (2002). Identificação dos Perfis Genético e Somatotípico que Caracterizam Atletas de Voleibol Masculino Adulto de Alto Rendimento no Brasil. F\&PJ 1:12-20.

25. Fernandes Filho J, João A (2002). Identificação do perfil genético, somatotípico e psicológico das atletas brasileiras de ginástica olímpica feminina de alta qualificação espor-tiva. F\&PJ 1:12-20.

26. Fernandes Filho J, Pável DAC (2004). Identificação dos perfis dermatoglífico, somatotípico e das qualidades físicas básicas de atletas de alto rendimento na modalidade de natação em provas de meio-fundo e fundo. F\&PJ 3:18-28.
27. Ferreira AAM. (2004) Perfil dermatoglíico, somatotípico e das qualidades físicas de atletas brasileiros de corrida de orientação de alto rendimento. Dissertação (Mestrado em Ciência da Motricidade Humana). Rio de Janeiro: Universidade Castelo Branco.

28. Fukunaga $T$, Ichinose $Y$, Ito $M$, Kanehisa H, Kawakami Y (2000). In vivo estimation of contraction velocity of human vastus lateralis muscle during "isokinetic" action. $J$ Appl Physiol 88:851-856.

29. Heath BH, Carter JE (1966). A comparison of somatotype methods. Am J Physiol Anthop 24:87-99.

30. Heath BH e Carter JE (1990). Somatotyping development and applications. New York: Cambridge University Press.

31. Silva Dantas PM, Fernandes Filho J (2002). Identificação dos perfis, genético, de aptidão física e somatotipico que caracterizam atletas masculinos, de alto rendimento, participantes do futsal adulto, no Brasil. F\&PJ 1:28-36.

32. Silva Dantas P. (2004) Relação entre estado e predisposição genética no futsal brasileiro. Tese (Doutorado em Educação Física). Natal: Universidade Federal do Rio Grande do Norte.

33. Thomas JR, Nelson JK (2002). Métodos de Pesquisa em Atividade Física. Porto Alegre: Artmed.

34. Wilmore JH, Costill DL (2001). Fisiologia do Esporte e do Exercício. São Paulo: Manole.

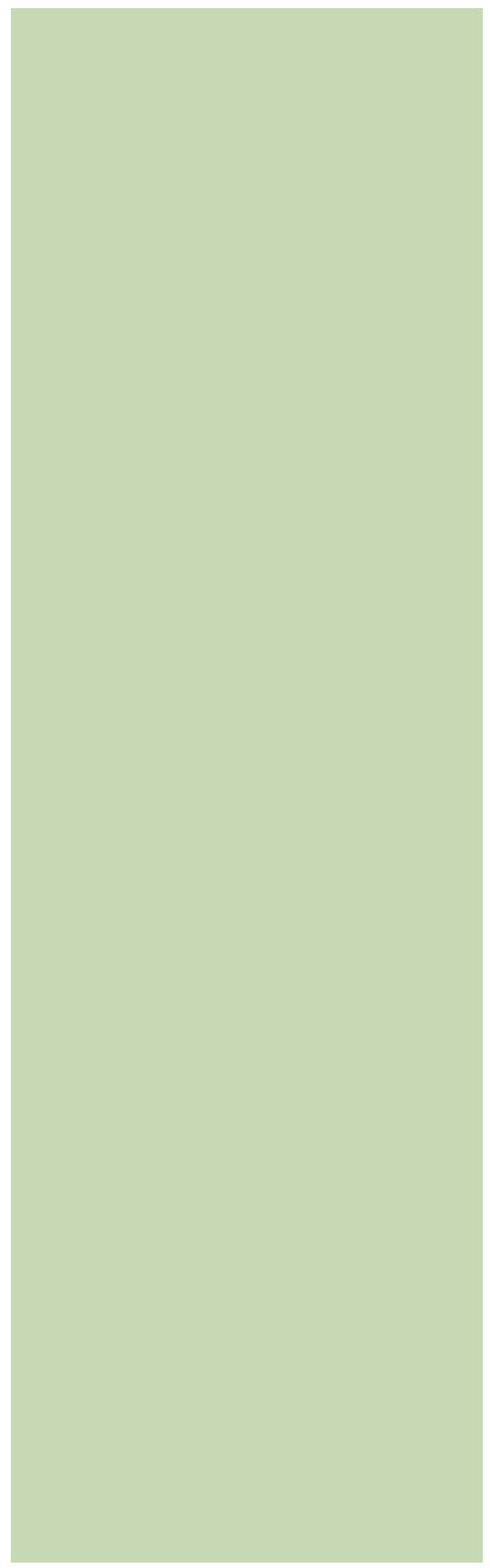

